\title{
Bilateral Adrenal Hemorrhage in a 6-Day-Old Neonate Presenting with Hematuria of 2 Days Duration: Case Report
}

This article was published in the following Dove Press journal: International Medical Case Reports Journal

\section{Tsion Tilahun \\ Gezahegn Diriba \\ Melkamu Berhane (D)}

Department of Pediatrics and Child Health, Jimma University, Jimma, Ethiopia
Background: The adrenal gland of newborns is susceptible to hemorrhage because of its relative larger size and high vascularity. Adrenal hemorrhage is bilateral in $10 \%$ of the cases. Neonates with adrenal hemorrhage present with anemia, jaundice, abdominal mass, and, rarely, adrenal insufficiency.

Case Detail: A 6-day-old macrosomic neonate presented with hematuria of 2 days duration. Delivery was difficult. Abdominal ultrasound demonstrated bilateral suprarenal echo complex mass (right $4.3 \mathrm{~cm}$ by $2.2 \mathrm{~cm}$ and left $4 \mathrm{~cm}$ by $2 \mathrm{~cm}$ ) and abdominal CT scan showed bilateral hypo-dense non-enhancing fluid-attenuated suprarenal gland masses (right $4.3 \mathrm{~cm}$ by $2.5 \mathrm{~cm}$ and left- $3.9 \mathrm{~cm}$ by $2.4 \mathrm{~cm}$ ).

Conclusion: Adrenal hemorrhage should be considered and looked for in at-risk newborns like those with macrosomia, overwhelming sepsis, and perinatal asphyxia as well as those with bleeding diathesis. Although rare, hematuria is one of the clinical features of adrenal hemorrhage in neonates. Asymptomatic neonates with adrenal hemorrhage may not need any intervention except close follow up.

Keywords: adrenal hemorrhage, neonate, haematuria, case report

\section{Introduction}

Adrenal hemorrhage is more common in neonates than children or adults due to the relatively large size and increased vascularity of the adrenal glands in fetuses and neonates. The incidence ranges from 1.7 to 2.1 per 1000 births but the real occurrence is probably higher as bleeding may remain asymptomatic. ${ }^{1}$

Adrenal hemorrhage is commonly seen in male neonates; $90 \%$ of the cases are unilateral and $75 \%$ of the cases occur on the right side. ${ }^{2}$ Since the right adrenal vein drains directly into the inferior vena cava, compression of the right adrenal gland between the liver and the spine induces venous pressure changes and hence increases the risk of hemorrhage. ${ }^{1}$ We hereby report a 6 -day-old male neonate with bilateral adrenal hemorrhage who presented with hematuria.

\section{Case Presentation}

A 4-day-old male neonate born to a Para-IV mother after 9 months of amenorrhea Department of Pediatrics and Child Health, Jimma University, P.O. Box: 378 , Jimma, Ethiopia

Tel +25I 966880698

Email tsiyohany@gmail.com presented with intermittent reddish discoloration of urine and occasionally passing frank blood per urethra. The mother had antenatal care visits 8 times and there was no problem identified during pregnancy. Delivery was by spontaneous vaginal delivery after 
a duration of labor of $9 \mathrm{hrs}$ and rupture of membrane of $6 \mathrm{hrs}$. The delivery was difficult associated with shoulder dystocia. First and fifth minute APGAR scores were 7 and 9 respectively and birth weight was $5000 \mathrm{gm}$. The neonate did not receive vitamin K after birth. The history was otherwise unremarkable.

On physical examination, PR was $112^{\prime}$, RR $42^{\prime}$, temperature $36.7^{\circ} \mathrm{C}$ and $\mathrm{spO}_{2} 96 \%$; weight was 4600 gm and head circumference $35.5 \mathrm{~cm}$. The abdomen was flat, with no palpable mass or organomegaly, and no area of tenderness, bruise, skin discoloration, or sign of fluid collection. Both testicles were palpable in the scrotum and there was no scrotal swelling or discoloration but frank, bright red blood was noticed on the diaper. He had no pallor, skin discoloration,or petechiae and all primitive reflexes were intact.

With an initial diagnosis of term, macrosomia, large for gestational age, and hematuria secondary to rule out congenital Wilms' tumor, he was investigated with CBC (WBC 11,190/ $\mu \mathrm{L}$, hematocrit $69 \%$, and platelets $244,000 / \mu \mathrm{L}$ ), urinalysis (blood trace, RBC 3-7/HPF) and renal function test (creatinine 0.84, BUN 20.4). Abdominal ultrasound showed bilateral suprarenal echo complex mass (right $4.3 \mathrm{~cm}$ by $2.2 \mathrm{~cm}$ and left $4 \mathrm{~cm}$ by $2 \mathrm{~cm}$ ) and abdominal CT scan showed bilateral hypo-dense non-enhancing fluid-attenuated suprarenal gland masses (right $4.3 \mathrm{~cm}$ by $2.5 \mathrm{~cm}$ and left $3.9 \mathrm{~cm}$ by $2.4 \mathrm{~cm}$ ) with a final conclusion of bilateral suprarenal gland collection likely hemorrhage (Figure 1). We did not do additional investigations like serum electrolytes (as the patient was clinically stable) and cortisol or ACTH stimulation tests (as these tests were not available in the setting). The neonate was admitted to the neonatal intensive care unit (NICU) for inpatient follow up and we did not provide any treatment apart from follow up as he did not have any additional symptom apart from the hematuria.

During the follow up, subsequent hematocrit measurements showed values of $50 \%$ and $54 \%$ on the 7 th and 8 th days of life respectively. He stayed in our hospital for 3 days for observation. During his 3-day stay in the NICU, the urinary complaint gradually subsided, his urine became clear, and he was passing urine adequately and doing well clinically. So, he was discharged with parental counseling on the natural course of the disease, the need for subsequent clinical and radiological follow up, and advice on when to return immediately.

Follow up evaluation 1 month after discharge showed a well growing infant with no remarkable finding; ultrasound showed bilateral hypo-echoic suprarenal masses decreasing in size (right $2.5 \mathrm{~cm}$ by $1.5 \mathrm{~cm}$ and left $1.9 \mathrm{~cm}$ by $1.8 \mathrm{~cm}$ ) and all other structures were normal.

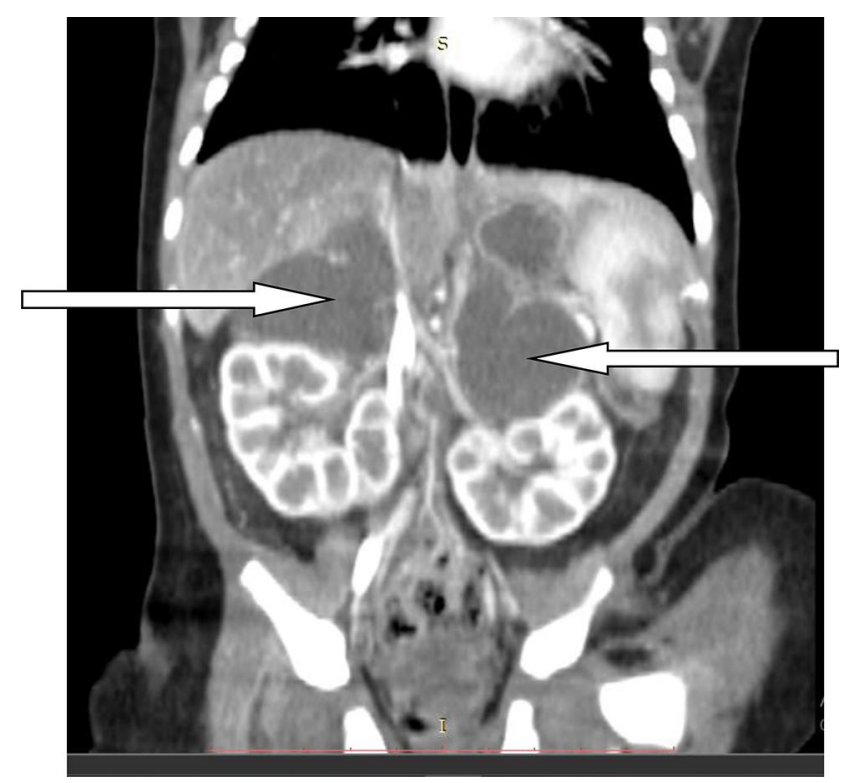

Figure I CT scan of abdomen reveals bilateral adrenal hemorrhage (arrows) in a 6-day-old neonate.

Follow up ultrasound done at 5 months of age demonstrated normal findings and the infant was doing well with no complaint.

\section{Discussion}

Adrenal hemorrhage is the most common cause of adrenal mass in neonates, $10 \%$ of the cases occurring bilaterally. ${ }^{2,3}$ Our case was among the few cases of bilateral adrenal hemorrhage.

Even if the etiology of neonatal adrenal hemorrhage might not be apparent, there are several possible risk factors which include maternal diabetes, macrosomia, perinatal asphyxia, overwhelming sepsis, prolonged labor, breech delivery, and instrumental delivery. Additional risk factors include bleeding disorders, anticoagulant administration, and child abuse. ${ }^{2,4}$ In our case, the possible identified risk factors were macrosomia and difficult delivery. In neonates with adrenal hemorrhage, the kidneys could be involved in several ways, one of which is vascular obstruction due to an extension of thrombosis in the adrenal vein into the renal vein, or a retrograde spread of thrombosis from the inferior adrenal artery into the renal artery which could in turn lead to different renal symptoms including hematuria. $^{4}$

The spectrum of presentation of adrenal hemorrhage ranges from asymptomatic to severe life-threatening intraabdominal hemorrhage and hypoadrenalism. Clinical findings also vary considerably from normal to palpable abdominal mass. $^{3}$ Additionally, anemia, unexplained jaundice, hematuria, 
or scrotal hematoma or swelling may be the presenting sign. ${ }^{2,4,6}$ Laboratory findings include anemia, hyponatremia, hyperkalemia, hypoglycemia, hypocortisolemia, eosinophilia, leukocytosis, hematuria, proteinuria, elevated adrenocorticotropic hormone, and elevated renin activity. ${ }^{2,4}$ In our case, apart from hematuria, there was no other symptom.

Initial diagnosis of an adrenal mass in a neonate is usually made by ultrasound whereas CT and MRI are helpful to rule out other possible differential diagnoses like congenital neuroblastoma, Wilm's tumor, hydronephrosis, cystic renal disease, and vascular thrombosis. ${ }^{3}$

Treatment of neonatal adrenal hemorrhage depends on the severity and associated illness. Infants with acute hemorrhage need urgent replacement of blood and administration of vitamin $\mathrm{K}$. Large doses of hydrocortisol should be given in all bilateral hemorrhages and in cases not responding adequately to blood or intravenous fluid administration. ${ }^{4}$ Laparotomy is indicated when hemorrhage seems to be continuing, especially when the exact site of hemorrhage is in doubt. ${ }^{4}$ In our case, we did not do any intervention apart from observation and follow up since the infant was clinically and hemodynamically stable.

Adrenal hemorrhages usually resolve within 3 months and may take up to 9 months in ultrasonographic followup. ${ }^{1}$ Hence, the usual follow-up time for the resolution of the hemorrhages should be within 90 days and neuroblastoma should be suspected if the mass is not resolved after this period. ${ }^{5}$ After resolution of the hemorrhages, calcification may be seen in one-third of children. ${ }^{1}$ The natural course of the hemorrhage in our case was also in line with this, completely resolving in 5 months.

\section{Conclusion}

Adrenal hemorrhage should be considered and looked for in at-risk newborns like those with macrosomia, overwhelming sepsis, and perinatal asphyxia as well as those with bleeding diathesis. Though rare, hematuria is one of the clinical features of adrenal hemorrhage in neonates. Close monitoring and regular follow up clinically and sonographically could suffice in asymptomatic cases of neonatal adrenal hemorrhage.

\section{Ethical Consideration}

As this was a case report, institutional approval for publication was not required. However, the parents of the infant have given us their verbal consent to publish the case and the images and it was approved by Jimma University ethics committee. The verbal consent was witnessed, and details are stored on a file.

\section{Acknowledgment}

We would like to thank the parents for giving us their consent to report the case.

\section{Disclosure}

The authors declare that they have no conflicts of interest related to this work.

\section{References}

1. Demirel N, Baş AY, Zenciroğlu A, et al. Adrenal bleeding in neonates: report of 37 cases. Turk J Pediatr. 2011;53(1):43-47.

2. Zessis NR, Nicholas JL, Stone SI. Severe bilateral adrenal hemorrhages in a newborn complicated by persistent adrenal insufficiency. Endocrinol Diabetes Metab Case Rep. 2018;2018:2-6.

3. Hill EE, Williams JA. Massive adrenal haemorrhage in the newborn. Arch Dis Child. 1959;34(174):178-182. doi:10.1136/adc.34.174.178

4. Black J, Williams DI. Natural history of adrenal haemorrhage in the newborn. Arch Dis Child. 1973;48(3):183-190. doi:10.1136/ adc.48.3.183

5. MunganAkın İ, Tabel Y, Güngör S, et al. Neonatal adrenal hemorrhage as a complication of traumatic vaginal delivery: diagnosis and follow-up with ultrasonography. Ann Med Res. 2008;15(2):109-111.

6. Bhatt S, Ahmad M, Batra P, et al. Neonatal adrenal hemorrhage presenting as "Acute Scrotum" - looking beyond the obvious: a sonographic insight. $J$ Ultrasound. 2017;20(3):253-259. doi:10.1007/s40477-017-0248-3

\section{Dovepress}

\section{Publish your work in this journal}

The International Medical Case Reports Journal is an international, peer-reviewed open-access journal publishing original case reports from all medical specialties. Previously unpublished medical posters are also accepted relating to any area of clinical or preclinical science. Submissions should not normally exceed 2,000 words or 4

published pages including figures, diagrams and references. The manuscript management system is completely online and includes a very quick and fair peer-review system, which is all easy to use. Visit http://www.dovepress.com/testimonials.php to read real quotes from published authors. 